evident that, apart from the convulsions, which in this case were probably the actual cause of death, the patient had suffered from acute laryngeal obstruction.

The striking features of this case, which occurred in 1934, were the speed with which the emphysema developed and the fact that by the time it was first noticed it was already too late to do much about it, and that the emphysema appeared after the introduction of an endotracheal tube. The whole operation was completed in less than 20 minutes, and the anaesthetic was administered by an experienced anaesthetist.

\section{Case II}

A coloured boy aged 11 years was admitted to hospital with a diagnosis of " bronchiectasis-for investigation," with a view to surgical treatment of the condition. He had suffered from a cough with foul sputum for some years as well as frequent colds of long duration, and two febrile attacks of more severity which were thought to be bronchopneumonia. On admission he was of average size for his age, with early clubbing of the fingers. Clinical examination of the chest revealed showers of rales over the right lung and occasional rhonchi. He expectorated daily $4 \mathrm{oz}$. of foul-smelling sputum. The plain radiograph suggested a cystic bronchiectasis in three lobes of the right lung.

In view of the foul sputum, postural drainage was carried out for several weeks, before attempting bronchography, in an endeavour to clear the bronchial tree. When it was finally decided to carry out the examination the sputum had been reduced to $1 \mathrm{oz}$. daily. His age and temperament were considered to be unsuitable for successful bronchography under local analgesia, and so a general anaesthetic was given.

On Feb. 22, 1944, hè was given morphine gr. 1/12 and atropine sulphate gr. 1/100 as premedication. The child was then anaesthetized with $\mathrm{CHCl}_{3}$ and oxygen. The induction was not uneventfulsputum proving troublesome and cyanosis difficult to eliminate. A Magill endotracheal tube was therefore passed under direct vision and the anaesthetic continued. Cyanosis was still prominent, and on one occasion the pulse faded; oxygen was liberally administered in an attempt to combat this. About 15 minutes after the induction the anaesthetist considered the patient to be more or less settled, and as the cyanosis had improved he felt that the bronchogram could now be made. It was, however, plain that the condition of the patient was not altogether satisfactory. It had been our recent custom in small children receiving a general anaesthetic for bronchography to inject the lipiodol through a fine Monaldi catheter inserted into the lumen of the endotracheal tube. This catheter was so small that, while allowing a flow of lipiodol, it encroached but little upon the airway. This method has not caused us any anxiety. At the moment when the oil was about to be instilled it was noticed that the child's neck was grossly deformed, there being complete loss of the angle between the chin and the neck, and that the neck was alarmingly ballooned right down to the clavicles. Palpation suggested deep emphysema, little or no subcutaneous emphysema being observed. The child was again cyanotic; the $\mathrm{CHCl}_{3}$ was discontinued at once and the oxygen flow increased. A serum needle, which was fortunately lying on the bronchography trolley, was inserted into the left side of the neck about two fingerbreadths above the clavicle. No diminution in the size of the neck occurred, and the child's condition appeared to be critical. A 20-c.cm. Record syringe was then applied to the needle, but the absolute resistance of the plunger to withdrawal suggested that the needle-point had not reached an air-containing fascial space. The needle was then inserted more deeply until the plunger could be withdrawn with ease. Aspiration was started, the thumb being placed over the hilt an disconnecting the syringe. During the first minute or so there did not seem to be an appreciable reduction in size, but as the plunger's easy withdrawal suggested the continual aspiration of air, the process was continued and the swelling began to recede. At this point a two-way adapter had been procured: the reduction was then more rapid and the aspiration on a better basis. When the contour of the neck seemed almost normal and the condition of the patient much improved, the syringe was disconnected, but the needle was left in situ. Within a few seconds the neck ballooned up once again. The process of aspiration was repeated, and the neck returned to reasonable dimensions once more. On disconnecting the syringe a further ballooning occurred. Again aspiration was performed for a period of about 15 minutes, and on this occasion on disconnecting the syringe ballooning did not recur. The oxygen administered in this last 15 -minute period was appreciably reduced in amount. It has been estimated that, in all, aspiration was carried out for a full half-hour. While waiting to ensure that no further gross emphysema occurred a radiograph was taken, as the child had been anaesthetized in the $x$-ray room. The radiograph shows the needle in situ and emphysema to be present in the neck-although the neck appeared normal to palpation.

The child made an uneventful recovery from this operation with no further treatment; but a full course of sulphathiazole was given as a precaution against infection of the fascial planes in view of the foul expectoration.

On examination of the anaesthetic apparatus it was apparent that the expiratory valve was not working at the maximum of efficiency and that the pressure of gases in the pharynx must have been unduly high. The anaesthetic was administered by an anaesthetist of skill and experience in the procedure.

\section{Summary and Conclusions}

Two cases of massive surgical emphysema which occurred during the administration of general anaesthesia are recorded. The common features are: the rapid onset of the condition; the alarming rate of spread; and that in both cases oxygen was liberally administered to combat an emergency-convalsions in one and cyanosis in the other. It is suggested that incision or the mere insertion of needles into the superficial tissues is not sufficient to control the emphysema, and that aspiration is necessary; that if the cause remains the emphysema returns with alarming rapidity when aspiration of air is discontinued. Finally, if aspiration is attempted the needle may have to be passed through the deep fascia of the neck.

In both these cases it is probable that the condition was caused by a minute perforation of the pharyngeal mucosa, through which gas under pressure, was forced into the fascial planes of the neck and mediastinum. It is therefore conceivable that, once such a process has been started, it might continue, even after the primary cause has been removed, if the patient develops an obstruction to the airway and struggles for breath.

\section{REFERENCES}

Georg, C. (1917). Amer. J. Surg. (Anaes. Supp.), 31, 77.

Luke, H. C. (1913). Surg. Gynec. Obstet., 16, 204.

Macklin, C. C (1937) Canad med. Ass, J, 36, 414

Marcotte, R. J.. Phillips, F. J., Adams, W. E., and Livingstone, H. (1940).

J. thorac. Surg.i 9, 346. St. J. Med., 15, 93.
Woolsey, W. C. (19i2). N.Y. St.

\section{AN UNUSUAL CASE OF GENERALIZED TUBERCULOSIS}

BY

\section{W. H. MYLECHREEST, M.B., B.Ch. Major, R.A.M.C. AND}

I. M. SCOTT, M.B., Ch.B. Capt., R.A.M.C

The following case of generalized tuberculosis seems to us to be well worth recording.

\section{Case History.}

An American seaman aged 26 was suddenly taken ill on Nov. 22, 1943 , complaining of headache and chills. He ran a continuous fever from the onset to his death in March, 1944. During the course of the illness, which was difficult to diagnose, he developed erythema nodosum. The interest of the case is in the $x$-ray and post-mortem findings, which are as follows:

Summary of X-Ray Reports.-23/12/43:-Marked increase in left hilar shadow, continuous with a fan-shaped opacity spreading into left mid-lung zone; consistent with a localized area of consolidation and associated glandular enlargement. 30/12/43:- No appreciable change. 14/1/44:- Increase in size of opacity in left hilum and mid-lung zone; consistent with tuberculous infiltration and glandular enlargement of the type usually seen in children. 20/1/44:Increase in the spread. $9 / 2 / 44$ :- Increase in size and density, associated with widening of the mediastinum. The possibility of lymphadenoma or neoplasm, with secondary lung involvement, cannot be excluded. $21 / 2 / 44$ :- Slight increase of the glandular enlargement to the right of the midline.

Post-mortem Examination.-Lungs:-There was approximately half a pint of deep-yellow fluid in the left pleural cavity. There were adhesions at the base on the left side, and a large breakingdown caseous peribronchial lymph gland in the left side of the mediastinum approximately the size of a tangerine. The lower lobe of the left lung was solid, very little caseation being apparent. There were some scattered tubercles throughout the rest of the left lung and also the right lung. The condition in the left lower lobe seemed to be a direct spread from the affected peribronchial gland. Trachea and bronchi were not involved in the disease process. Heart:There was a .large amount of fibrinous pericarditis. Numerous tubercles (up io $1 / 4$ in. in diameter) were present in the pericardium: these did not extend into the myocardium. However, a solitary tubercle (approximately $1 / 2$ in. in diameter) was found in the myocardium of the right ventricle. There was healthy myocardium between this tubercle and the pericardium. The valves were normal 
in appearance, as were the aorta and coronary arteries. The liver was enlarged, and was the seat of many tubercles. These varied in size from $1 / 4$ in. to $1 / 2$ in., and showed a surprising lack of caseation. They were in several cases surrounded by necrotic liver tissue and there was little or no fibrosis around the tubercles. The spleen was enlarged to about four times the normal size and was congested. Here again were seen many tubercles similar in size and appearance to those in the liver. There was a fibrinous perisplenitis. The kidneys showed several small tubercles scattered through the organs. The pancreas appeared normal, though there was an enlarged semicaseous lymph gland near the head. The suprarenals were normal. The oesophagus, stomach, and intestines were also normal in appearance. There was little free fluid in the abdominal cavity, and only an occasional small tubercle was present in the peritoneum.

Microscopical Examination.-Smears taken from the peribronchial lymph gland and from a tubercle in the liver showed acid alcoholfast bacilli. Sections of the tubercle in the myocardium revealed a central area of early caseation, surrounded by-endothelioid cells, histiocytes, and small round cells. Scanty giant cells of the Langhans type were present. Acid alcohol-fast bacilli were also found.

\section{Comments}

The heart and pericardium are seldom involved in miliary tuberculosis, and although solitary tubercles in the myocardium have been described, they are quite exceptional. Tuberculosis of the pericardium is not uncommon, and is due in most cases to a backward infection by the lymphatics from a tuberculous gland. To quote Muir's Pathology (4th edition, 1940): "At an early stage tubercles are formed, but these soon lead to inflammatory reaction and become covered with fibrin. The disease may thus appear like an ordinary fibrinous pericarditis, and its real nature may be discovered only on microscopical examination." In this case, however, there were, as already cescribed, many large and very obvious tubercles. The lesion in the myocardium (1/2 in. in diameter) was discrete and presumably blood-borne, as no connexion between it and the pericardium could be traced either macroscopically or microscopically. The condition in the liver, too, is very rare. To quote the above author again: "The presence of large almost tumour-like tuberculous masses in the liver has been recorded in a few cases, but the occurrence is very rare."

The disease in this case would appear to have been a bloodstream infection with a ? primary focus in the peribronchial lymph gland. The size of the tubercles and the absence of macroscopic caseation in the tubercles in the various affected organs argue a comparatively high resistance on the part of the patient, and in some way account for the lack of clinical $s$ 'gns which, until within a few weeks of his death, consisted merely of a persistent pyrexia and the early erythema nodosum.

We wish to thank Col. W. D. Arthur, M.B.E., and Lieut.-Col. W. L. Ackerman for their help and permission to forward this paper.

\section{Medical Memoranda}

\section{Local Analgesia for Adult Gircumcision}

No claims for originality are made when a technique of local block for adult circumcision is described ; but at the same time the textbooks do not stress this method as being a technique of choice for this operation. Under certain conditions it is definitely the most satisfactory anaesthesia. If a general anaesthetic is employed circumcision in the adult demands a much deeper level of anaesthesia than is of ten realized. For instance, a "shot of pentothal" will not do ; a large dose of this barbiturate is not sufficient in most cases. The advantages claimed for local block are speed for the surgeon in the organization of a clinic, a high degree of haemostasis, and the use of a very small quantity of an inexpensive fluid. And the patient is not subjected to the risks of a general anaesthetic, small though these are.

Recently, local block for adult circumcision was carried out in 41 cases at a military. hospital in India, on British and Indian troops. The cases were largely drawn from the venereal disease department, but there were cases of non-venereal balanitis and phimosis also. During this series, admittedly small, no other technique was used, although there were slight variations in the method. Sepsis was not considered to be a contraindication, and a few of the cases were in such a condition that the operation was confined to a "dorsal slit."

Of course the soldier is the ideal subject for all forms of block analgesia, provided that they are successful. Reports of incomplete anaesthesia and the consequent suffering very quickly spread round a ward. Patients then arrive at the theatre in the worst possible state of anxiety for a local. Therefore as soon as any pain is felt it is essential for something to be done, and the best is a very light inhalation, open ether being satisfactory. One case in this series was so treated, and the confidence of the wards was not upset.

The patients were sent to the theatre at the same time, and were anaesthetized in a batch if the number on the list did not exceed four. One patient was circumcised after a lapse of an hour, and there was no falling off in the standard of analgesia. The question of premedication is simple in these cases. From this series it was decided that, whether morphine was given or not, the results were the same as regards the analgesia, but a greater tranquillity of mind was present in the premedicated. But we were dealing with trained soldiers, and it is suggested that in the case of a civil clinic all cases be premedicated. In order to cut down the number of stretcherbearers which would be required-since the ward which provided these cases was a long distance from the theatre-the patients walked to the anaesthetic room. At the beginning of the series all cases were given morphine gr. 1/3 intravenously on arrival. Then the local analgesic was injected, novutox being the agent, and the dose within the limits of 6 to $8 \mathrm{c} . \mathrm{cm}$. A $10-\mathrm{c} . \mathrm{cm}$. syringe fitted with a hypodermic needle was used. The first injection was made into the base of the penis on the dorsal surface in the midline, and the area on each side of this puncture was infiltrated as far as the lateral border of the organ. Approximately $4 \mathrm{c.cm}$. of agent would be used over this area. A second puncture was then made into the ventral surface in the midline, and this area was similarly infiltrated to meet the lateral boundaries of the first injection. Less solution would be required here, since the previous infiltration had included the lateral borders. But before the needle was removed at least $1.5 \mathrm{c.cm}$. was inserted into the midline of the ventral surface. This was most important, as in any case of imperfect anaesthesia the frenum is the sensitive structure. After the infiltration was completed a period of at least ten minutes was allowed to elapse before the operation was started. When premedication was abandoned, about half-way through the series, the patients were allowed to walk back to the ward, accompanied by an orderly, and every one arrived there none the worse for the experience.

It has been suggested that the efficient haemostasis provided by the local block might lead to post-operative haemorrhage ; this did not happen. No doubt the haemostasis is produced by the mechanical compression of the vessels by the ring of swelling resulting from the infiltration. There were no ill effects.

I am indebted to Major J. D. Wilson. R.A.M.C., surgical specialist to the hospital where these cases were treated, for his interest and co-operation in the anaesthesia.

\section{H. L. Rogerson, M.R.C.S., L.R.C.P.,} Major, R.A.M.C.

\section{A Case of Sensitiveness to Strychnine}

The following record seems worthy of publication not only for its rarity but for the moral lesson it affords.

\section{CASE RePort}

A married woman aged 42 came under my care in a busy panel practice recently, already certified as unfit for work on account of rheumatism, of which there was no objective evidence. Thinking that aperient treatment might be of value, I prescribed a bottle of mist. casc. co. Next day I was summoned urgently, to find that after one dose of the new medicine the patient had collapsed, and was pale and pulseless. No definite diagnosis occurred to me, although I toyed with the idea of belladonna poisoning in an aberrant form, since, as some may know, the mist. casc. co. of the 1943 Formulary contains the astonishing amount of $12 \frac{1}{2}$ minims of the tincture to the dose, whereas the 1939 mist. casc. co. contains only 3 minims, as I found on looking it up.

After some weeks of treatment with iron the patient asked for a tonic, intending to return to work. I prescribed the mist. phosp. $\bar{c}$ strych., of which the patient warily took only a teaspoonful, and within a few minutes suffered a similar though not so severe attack. On my arrival in haste $I$ had to defend myself against accusations of poisoning. Since the only item common to the two mixtures is strychnine, I was forced to the conclusion that this must be the cause of the attacks, and at the request of the patient obtained another opinion. My colleague rather sceptically injected intradermally $0.05 \mathrm{c.cm}$ of liq. strych, which caused an immediate wheal followed in a minute or two by a typical anaphylactic attack, which required the injection of adrenaline.

I have now equipped the patient with a written note to be shown to any future doctor. Inquiry among friends and chemists of wide experience has not brought to light a similar case.

D. R. C. Shepherd, M.R.C.P. 